\title{
Clinical efficacy and predictive biomarkers of ONC201 in H3 K27M-mutant diffuse midline glioma
}

Carl Koschmann ( $\nabla$ ckoschma@med.umich.edu )

Michigan Medicine

Abed Rahman Kawakibi

Michigan Medicine

Rohinton Tarapore

Oncoceutics (United States)

Sharon Gardner

NYU Langone Health

Chase Thomas

Michigan Medicine

Rodrigo Cartaxo

Michigan Medicine

Viveka Yadav

Michigan Medicine

Andrew Chi

NYU Langone Health

Sylvia Kurz

NYU Langone Health

\section{Patrick Wen}

Dana-Farber Cancer Institute

Isabel Arrillaga

Massachusetts General Hospital

Tracy Batchelor

Massachusetts General Hospital

Nicholas Butowski

University of California, San Francisco

Ashley Sumrall

Levine Cancer Institute

Nicole Shonka

University of Nebraska Medical Center

Rebecca Harrison

University of Texas MD Anderson Cancer Center, Houston, John De Groot 
The University of Texas MD Anderson Cancer Center

\section{Minesh Mehta}

Miami Cancer Institute

Yazmin Odia

Miami Cancer Institute

Matthew Hall

Miami Cancer Institute

Doured Daghistani

Miami Cancer Institute

Timothy Cloughesy

University of California Los Angeles https://orcid.org/0000-0002-8656-7483

Benjamin Ellingson

University of California Los Angeles

Michelle Kim

Michigan Medicine

Yoshie Umemura

Michigan Medicine

Hugh Garton

University of Michigan

Andrea Franson

Michigan Medicine https://orcid.org/0000-0002-5361-7683

\section{Patricia Robertson}

Michigan Medicine

Jonathan Schwartz

University of Nebraska Medical Center

\section{Bernard Marini}

Michigan Medicine https://orcid.org/0000-0003-0436-8948

\section{Manjunath Pai}

Michigan Medicine https://orcid.org/0000-0001-7119-5034

\section{Timothy Phoenix}

University of Cincinnati

\section{Sunjong Ji}

Michigan Medicine https://orcid.org/0000-0002-6516-2380

\section{Evan Cantor}

Michigan Medicine

\section{Zachary Miklja}

Michigan Medicine

\section{Brendan Mullan}

Michigan Medicine 


\section{Amy Bruzek}

Michigan Medicine

\section{Ruby Siada}

Michigan Medicine

Jessica Cummings

Michigan Medicine

\section{Stefanie Stallard}

Michigan Medicine

Kyle Wierzbicki

Michigan Medicine

Alyssa Paul

Michigan Medicine

lan Wolfe

Michigan Medicine

\section{Matthew Dun}

University of Newcastle https://orcid.org/0000-0002-9063-5370

\section{Jason Cain}

Hudson Institute of Medical Research https://orcid.org/0000-0003-3987-5894

\section{Li Jiang}

Dana-Farber Boston Children's Cancer and Blood Disorders Center

\section{Mariella Filbin}

Dana-Farber Boston Children's Cancer and Blood Disorders Center

\section{Pankaj Vats}

University of Michigan https://orcid.org/0000-0001-8441-7732

\section{Chandan Kumar-Sinha}

University of Michigan

\section{Rajen Mody}

Michigan Medicine

\section{Arul Chinnaiyan}

University of Michigan-Ann Arbor https://orcid.org/0000-0001-8743-7480

\section{Drew Pratt}

University of Michigan-Ann Arbor

\section{Sriram Venneti}

University of Michigan-Ann Arbor

\section{Guangrong Lu}

Oncoceutics

\section{Sabine Mueller}

University of California, San Francisco https://orcid.org/0000-0002-8216-9357

\section{Adam Resnick}


Children's Hospital of Philadelphia

Javad Nazarian

Children's National Health System

Sebastian Waszak

University of Oslo and Oslo University Hospital https://orcid.org/0000-0003-3042-9521

Joshua Allen

Oncoceutics

\section{Letter}

Keywords: Diffuse Midline Glioma, ONC201 Treatment, H3 K27M mutation, Telencephalic Gene Regulatory Network

Posted Date: September 10th, 2020

DOI: https://doi.org/10.21203/rs.3.rs-69706/v1

License: (c) (i) This work is licensed under a Creative Commons Attribution 4.0 International License. Read Full License 


\section{Abstract}

Patients with diffuse midline glioma (DMG) harboring H3 K27M mutation have no proven therapies beyond radiation. ONC201, a DRD2 antagonist and mitochondrial ClpP agonist, has induced early responses in patients with H3 K27M-mutant DMG. We performed an integrated pre-clinical and clinical assessment of ONC201 treatment, in order to define response rates in H3 K27M-mutant DMG patients and to clarify predictors of response. ONC201 was effective in murine H3 K27M-mutant gliomas with excellent CNS penetration and survival benefit. H3 K27M-mutant DMG patients treated with ONC201 on active clinical trials $(n=50)$ showed significant survival benefit in recurrent and non-recurrent settings, with multiple sustained responses. Tumor sequencing from treated patients demonstrates an EGFR/FOXG1driven telencephalic gene regulatory network that imparts a critical resistance phenotype to ONC201. Genetic and pharmacologic knockdown of EGFR in H3 K27M-mutant cell cultures results in improved sensitivity to ONC201 and reduced FOXG1 enhancer binding, suggesting possible future combinatorial opportunities.

\section{Main}

The histone mutation H3 K27M in diffuse midline gliomas (DMG) is associated with aggressive clinical behavior and overall median survival of 12.0 months $^{1-3}$. The H3 K27M mutation is most commonly found in midline central nervous system (CNS) structures in children and young adults ${ }^{4}$, where the thalamus and brainstem account for the majority of patients carrying the mutation ${ }^{1,5-7}$. We assessed the bloodbrain barrier (BBB) penetration of ONC201 in midline brain structures in non-tumor bearing mice. As previously shown ${ }^{8,9}$, oral ONC201 achieves concentrations in brain tissue that greatly exceed plasma concentrations (Fig. 1a). At 30 minutes after treatment with $15 \mathrm{mg} / \mathrm{kg}$ ONC201, all midline structures demonstrated micromolar $(\mu \mathrm{M})$ concentrations (Fig. 1a). To evaluate the in vivo efficacy of ONC201, we adopted a midline in utero electroporation (IUE) mouse model of H3 K27M- glioma ${ }^{10,11}$, in which tumors harboring dominant negative TP53, PDGFRA D842V, and H3F3A (H3.3) K27M mutations ("PPK") are generated (Fig. 1b-C). Early passage PPK neurospheres and human H3 K27M- mutant DMG cell cultures SF7761 and QCTB-R059 demonstrate ONC201 sensitivity with IC50 of 0.5, 3 and 4 uM respectively (Fig. 1d); while H3 K27M-mutant DIPG007 cells remained relatively resistant. Weekly ONC201 treatment of mice bearing PPK tumors (125mg/kg once a week) significantly extended survival ( $p=0.02$; median 107 vs. 77 days) with no observed toxicity (Fig. 1e).

In order to define response rates in H3 K27M DMG patients and to clarify the genomic and clinical predictors of response, we performed an integrated assessment of H3 K27M-mutant DMG patients treated with ONC201. The clinical analysis included patients with primary tumors in the thalamus or brainstem and confirmed H3 K27M mutation (inclusion/exclusion criteria in Extended Data Fig. 1). Overall survival (OS) is 28.1 months from diagnosis (median time from diagnosis to trial enrollment: 8.7 months) in a total of 50 H3 K27M-mutant DMG patients treated with ONC201 (27 thalamic and 23 brainstem), surpassing historical median OS of 12 months ( $\left.n=274^{12}, p<0.0001\right)$ (Extended Data Fig. 2a). 
In thalamic tumors, median OS has not been reached with a median follow-up of 18.5 months from diagnosis [ $n=27$, historical median OS: 14.5 months $\left(n=68^{12}, p=0.0001\right)$ ] (Fig. 2a). Median OS is 17.1 months for patients with brainstem tumors [ $n=23$; historical median OS: 11.9 months $\left(n=206^{12}\right.$, $\mathrm{p}=0.0002$ )] (Fig. 2a). In patients who initiated ONC201 following radiation but prior to recurrence, median OS has not been reached with a median follow up of 16.8 months from diagnosis [ 8 thalamic, 8 brainstem; historical median OS: 12.0 months ( $n=274), p=0.0008$ ] (Fig. 2b). In the recurrent setting, median OS is 7.9 months from recurrence $(n=34)$, compared to historical H3 K27M median OS of 5.5 months from recurrence ${ }^{13,14}(p=0.0029)$ (Fig. 2c).

In the post-radiation non-recurrent setting, median PFS is 20.1 months from diagnosis for thalamic patients ( $n=8$; median time on drug without progression: 11.7 months, median time from diagnosis to trial enrollment: 3.7 months) and 13.0 months from diagnosis for brainstem patients ( $n=8$; median time on drug without progression: 5.9 months, median time from diagnosis to trial enrollment: 3.7 months) (Fig. $2 \mathrm{~d})$. In the recurrent setting, median PFS for thalamic patients is 3.3 months from enrollment, similar to median PFS for brainstem patients of 3.9 months (Extended Data Fig. 2b). Median OS and PFS in ONC201-treated H3 K27M-mutant DMG patients are better than historical controls for all sub-groups, even when censoring patients who remain on-treatment (sensitivity analysis) upon follow- up (Extended Data Fig. 3).

These data show that H3 K27M-mutant patients have improved outcomes with ONC201 treatment, particularly those with thalamic and non-recurrent tumors at enrollment. Thalamic tumors with evaluable tumor imaging $(\mathrm{n}=21)$ demonstrated 1 complete response (CR) and 5 partial responses (PR) by RANO, with 4 additional $(<50 \%)$ tumor regressions (Extended Data Fig. 4). Radiographic responses observed in thalamic patients on ONC201 treatment remain durable [median 10.7 (1.3-45.1) months] (Extended Data Fig. 5). Serial cell-free tumor DNA (tDNA) analysis for $\mathrm{H} 3.3 \mathrm{~K}^{2} \mathrm{MM}^{15}$ in plasma and CSF demonstrated correlation with sustained clinical and radiographic response (Fig. 2e-f)

To explore the molecular attributes of H3 K27M-mutant DMG patients who respond to ONC201, we analyzed a single institution cohort (University of Michigan; $n=13$ ) where baseline tumor RNA-seq ${ }^{16-18}$ was compared to best response (percentage reduction in tumor area). Analysis of known DMG driver genes revealed baseline expression of EGFR and the cortical developmental transcription factor FOXG1 as the strongest biomarkers of resistance to ONC201 (Fig. 3a). Interestingly, EGFR has been shown to mediate oncogenic phenotypes in glioma through FOXG1 signaling in adult GBM cells ${ }^{19}$. Conversely, H3 K27M-mutant DMG patients with increased baseline expression of gene sets involved in mitochondrial function demonstrated improved radiographic response (Figure 3b); consistent with recent data showing that ONC201 binds and activates the mitochondrial protease ClpP in leukemia cells ${ }^{20}$. Gene coexpression, functional enrichment ${ }^{21}$, and gene regulatory network analysis ${ }^{22}$ revealed a telencephalic molecular phenotype and reduced mitochondrial function in FOXG1+-H3 K27M-mutant DMGs (Extended Data Fig. 6). The latter association is consistent with FOXG1 regulation of mitochondrial bioenergetics in 
mouse neural stem cells ${ }^{23}$ and primary glial cells ${ }^{24}$; possibly introducing a glioma-specific resistance phenotype to ONC201.

Examining treatment responses in our cohort further, patients who had low expression of EGFR and FOXG1 exhibited the largest reductions in tumor size on ONC201 treatment (Fig. 3c-d). No somatic alteration correlated with response (Fig. 3e). However, patients with co-occurring EGFR mutations did not respond to ONC201 (UMICH-006 and UMICH-017). UMICH-006, a nine-year-old patient with H3- K27M thalamic DMG with an activating EGFR pathway mutation (EGFR ${ }^{\mathrm{A} 289 \mathrm{~V}}$ ) and high EGFR/ FOXG1 tumor expression, never responded to ONC201 (Fig. 3f), and showed a spike in H3 K27M CSF tDNA variant allele fraction (VAF) at 6 months correlating with metastatic progression (Fig. 3g).

We next performed an analysis of human cancer cell lines from DepMap/PRISM ${ }^{25}$ to assess correlation of FOXG1 and EGFR expression (and other reported predictive makers ${ }^{26-28}$ ) with sensitivity to ONC201. CLPP expression was identified as the strongest predictor of ONC201 sensitivity at the pan- cancer level ${ }^{29}$. A glioma-specific analysis (glioma cell lines) revealed high FOXG1 expression levels at baseline as the third most significant predictor of sensitivity to ONC201 (Extended Data Fig. 7a-b). The other direct target of ONC201 response, DRD2 ${ }^{26-28}$, did not predict ONC201 response in either our patient cohort or DepMap/PRISM glioma cell atlas. Further analysis of the pan-cancer cell line atlas demonstrated that ONC201 resistance correlates with: (i) EGFR expression, (ii) EGFR dependency and (iii) EGFR inhibitor (EGFRi) sensitivity (Fig. 4a). Interestingly, previous data demonstrated that antipsychotics targeting DRD2 and EGFR inhibitors can work synergistically to target adult GBM cells (H3 K27M WT) ${ }^{30}$.

Lentiviral-mediated shRNA knockdown of EGFR in the ONC201-resistant H3 K27M primary cell culture DIPG007 resulted in a transition from EGFR to PDGFRA activation (Fig. 4b) and marked improvement in response to ONC201 (Fig. 4c). This is consistent with positive correlation of PDGFRA expression with ONC201 treatment response in our clinical cohort (Fig. 3a). Two ONC201-sensitive H3 K27M cell cultures (SF7761 and QCTB-R059) displayed additive benefit to treatment with ONC201 and the EGFRi osimertinib (Fig. 4d and Extended Data Fig. 7c). Previous work has shown EGFR to drive oncogenesis in GBM through a FOXG1-dependent gene regulatory network ${ }^{19}$. Treatment of H3 K27M cells with osimertinib resulted in no change in FOXG1 protein levels (Extended Data Fig. 7c) but did reduce FOXG1 binding at established ${ }^{19}$ enhancer loci (Fig. 4e,f).

We next explored the expression of EGFR and FOXG1 in non-tumor cells to see if they may influence ONC201 sensitivity either through the micro-environment or cell of origin. FOXG1 and EGFR is highly expressed in the developing and adult murine telencephalon (cortex) in comparison to midline structures (Extended Data Fig. 8). In human high-grade glioma single-cell ${ }^{31}$ and bulk mRNA-seq ${ }^{3}$, EGFR and FOXG1 expression is highest in adult H3 WT GBM tumor cells, and lowest in H3 K27M thalamic cells/tumors (Extended Data Fig. 9). This is consistent with the exceptional clinical responses seen in thalamic H3 K27M patients in our ONC201 cohort. 
In our integrated preclinical and clinical analysis, ONC201 demonstrates unprecedented clinical activity in H3 K27M-mutant DMG, with favorable clinical and correlate responses. The most notable and durable responses are found in thalamic patients with therapy initiated following radiation and prior to recurrence. As a potential limitation of our analyses, patients included are clinical trial patients with a favorable baseline KPS (median KPS: 80), which introduces a potential selection bias when comparing patient outcomes with historical control. Nevertheless, the improvements in survival and sustained radiographic responses suggest a true treatment effect and establish ONC201 as the first therapy to improve outcomes in H3 K27M beyond radiation and re-irradiation.

Furthermore, we establish EGFR pathway activation and FOXG1 expression as critical negative predictors of ONC201 response in H3 K27M-mutant DMG. Our data demonstrates that FOXG1 introduces a telencephalic gene regulatory network in H3 K27M-mutant DMGs that imparts a previously un-recognized resistance phenotype to ONC201. Future study will determine whether this phenotype is driven by reduced mitochondrial bioenergetics and whether ONC201-resistant H3 K27M-mutant DMGs (and H3 WT GBMs) will respond to combinatorial EGFR/FOXG1 targeting treatments.

\section{Declarations}

\section{ACKNOWLEDGMENTS:}

The authors thank the patients and their families for participation in this study.

\section{AUTHORS CONTRIBUTIONS:}

The methodology and supervision were carried out by C.K; A.R.K, R.S.T, J.N, S.M.W, J.E.A, C.K interpreted preclinical and clinical data; A.R.K, S.M.W, C.K wrote the manuscript and the manuscript was reviewed and edited by all co-authors; S.G, A.C, S.C.K, P.Y.W, I.A, T.T.B, N.A.B, A.S, N.S, R.A.H, J.D, M.M, Y.O,

M.D.H, D.D, T.F.C, B.M.E, M.M.K, Y.U, S.M, C.K carried out the clinical investigations. R.S.T, G.L, J.E.A provided and reviewed clinical data; H.G, A.F, P.L.R, J.S, B.L.M, M.P.P, E.C, A.P, I.W, P.V, C.K-S, R.M, A.C, D.P, S.V interpreted and curated clinical data, C.T, R.C, V.N.Y, S.J carried out in vitro and in vivo experiments and analyzed data; S.M.W performed and interpreted computational analyses; T.N.P, Z.M, B.M, A.K.B, R.S, J.R.C, S.S, K.W M.D.D, J.E.C, L.J, M.G.F, A.R, J.N, S.M.W, generated and interpreted genomic and preclinical data.

\section{FUNDING:}

CK is supported by NIH/NINDS K08-NS099427-01, the University of Michigan Chad Carr Pediatric Brain Tumor Center, the Evans family, the Chad Tough Foundation, Catching up With Jack, the Prayers from Maria Foundation, the U CAN-CER VIVE Foundation, the Morgan Behen Golf Classic, and the DIPG 
Collaborative. The PEDS-MIONCOSEQ study was supported by grant 1UM1 HG006508 from the National Institutes of Health Clinical Sequencing Exploratory Research Award (PI: Arul Chinnaiyan). Clinical trials were supported by Oncoceutics, Inc. and NIH (CA192427) grant to JEA.

\section{DISCLOSURES:}

RST, GL, JEA are employees and shareholders of Oncoceutics Inc.

MM holds a BOD position on Oncoceutics with stock options, and has received honoraria for consulting relationships with Mevion, Karyopharm, Tocagen, Astra-Zeneca, Blue Earth Diagnostics, Celgene, and Abbvie.

\section{DATA AVAILABILITY STATEMENT:}

The datasets generated during and/or analyzed during the current study are available from the corresponding author on reasonable request.

\section{References}

1. Feng, J., et al. The H3.3 K27M mutation results in a poorer prognosis in brainstem gliomas than thalamic gliomas in adults. Hum Pathol 46, 1626-1632 (2015).

2. Karremann, M., et al. Diffuse high-grade gliomas with H3 K27M mutations carry a dismal prognosis independent of tumor location. Neuro Oncol 20, 123-131 (2018).

3. Mackay, A., et al. Integrated Molecular Meta-Analysis of 1,000 Pediatric High-Grade and Diffuse Intrinsic Pontine Glioma. Cancer Cell 32, 520-537 e525 (2017).

4. Solomon, D.A., et al. Diffuse Midline Gliomas with Histone H3-K27M Mutation: A Series of 47 Cases Assessing the Spectrum of Morphologic Variation and Associated Genetic Brain Pathol 26, 569-580 (2016).

5. Schreck, C., et al. Incidence and clinicopathologic features of H3 K27M mutations in adults with radiographically-determined midline gliomas. J Neurooncol 143, 87-93 (2019).

6. Wang, L., et al. H3 K27M-mutant diffuse midline gliomas in different anatomical locations. Hum Pathol 78, 89-96 (2018).

7. Miklja, Z., et al. Molecular profiling and targeted therapy in pediatric gliomas: review and consensus recommendations. Neuro Oncol (2019).

8. Allen, J.E., et al. Dual inactivation of Akt and ERK by TIC10 signals Foxo3a nuclear translocation, TRAIL gene induction, and potent antitumor effects. Sci Transl Med 5, 171 ra117 (2013).

9. Arrillaga-Romany, I., et al. Biological activity of weekly ONC201 in adult recurrent glioblastoma patients. Neuro Oncol 22, 94-102 (2020). 
10. Patel, S.K., et al. Generation of diffuse intrinsic pontine glioma mouse models by brainstem- targeted in utero electroporation. Neuro Oncol 22, 381-392 (2020).

11. Miklja, , et al. Everolimus improves the efficacy of dasatinib in PDGFRalpha-driven glioma. $J$ Clin Invest (2020).

12. Pratt, , et al. Circumscribed/non-diffuse histology confers a better prognosis in H3K27M-mutant gliomas. Acta Neuropathol 135, 299-301 (2018).

13. Mackay, A., et al. Molecular, pathological, radiological, and immune profiling of non-brainstem pediatric high-grade glioma from the HERBY phase II randomized trial. Cancer cel/ 33, 829-842. e825 (2018).

14. Mueller, S., et al. A pilot precision medicine trial for children with diffuse intrinsic pontine gliomaPNOC003: A report from the Pacific Pediatric Neuro-Oncology Consortium. International journal of cancer 145, 1889-1901 (2019).

15. Stallard, S., et al. CSF H3F3A K27M circulating tumor DNA copy number quantifies tumor growth and in vitro treatment response. Acta neuropathologica communications 6, 1-4 (2018).

16. Koschmann, C., et al. Clinically integrated sequencing alters therapy in children and young adults with high-risk glial brain tumors. JCO Precision Oncology 2, 1-34 (2018).

17. Mody, R.J., et al. Integrative clinical sequencing in the management of refractory or relapsed cancer in youth. Jama 314, 913-925 (2015).

18. Miklja, Z., et al. Molecular profiling and targeted therapy in pediatric gliomas: review and consensus recommendations. Neuro-oncology 21, 968-980 (2019).

19. Liu, F., et al. EGFR mutation promotes glioblastoma through epigenome and transcription factor network remodeling. Molecular cell 60, 307-318 (2015).

20. Ishizawa, J., et al. Mitochondrial ClpP-Mediated Proteolysis Induces Selective Cancer Cell Lethality. Cancer Cell 35, 721-737 e729 (2019).

21. Szklarczyk, D., et al. STRING v11: protein-protein association networks with increased coverage, supporting functional discovery in genome-wide experimental datasets. Nucleic Acids Res 47, D607D613 (2019).

22. Janky, , et al. iRegulon: from a gene list to a gene regulatory network using large motif and track collections. PLoS Comput Biol 10, e1003731 (2014).

22. Bulstrode, $\mathrm{H}$., et al. Elevated FOXG1 and SOX2 in glioblastoma enforces neural stem cell identity through transcriptional control of cell cycle and epigenetic regulators. Genes Dev 31, 757-773 (2017).

23. Pancrazi, L., et al. Foxg1 localizes to mitochondria and coordinates cell differentiation and bioenergetics. Proc Natl Acad Sci U S A 112, 13910-13915 (2015).

24. $\mathrm{Yu}$, , et al. High-throughput identification of genotype-specific cancer vulnerabilities in mixtures of barcoded tumor cell lines. Nature biotechnology 34, 419 (2016). 
25. Madhukar, N.S., et al. A Bayesian machine learning approach for drug target identification using diverse data types. Nat Commun 10, 5221 (2019).

26. Allen, E., et al. Discovery and clinical introduction of first-in-class imipridone ONC201. Oncotarget 7, 74380-74392 (2016).

28. Prabhu, V.V., et al. Dopamine Receptor D5 is a Modulator of Tumor Response to Dopamine Receptor D2 Antagonism. Clin Cancer Res 25, 2305-2313 (2019).

29. Corsello, S.M., et al. Discovering the anticancer potential of non-oncology drugs by systematic viability profiling. Nature Cancer 1, 235-248 (2020).

30. Li, J., et al. Genome-wide shRNA screen revealed integrated mitogenic signaling between dopamine receptor D2 (DRD2) and epidermal growth factor receptor (EGFR) in glioblastoma. Oncotarget 5, 882 (2014).

31. Filbin, M.G., et al. Developmental and oncogenic programs in H3K27M gliomas dissected by singlecell RNA-seq. Science 360, 331-335 (2018).

\section{Figures}

a

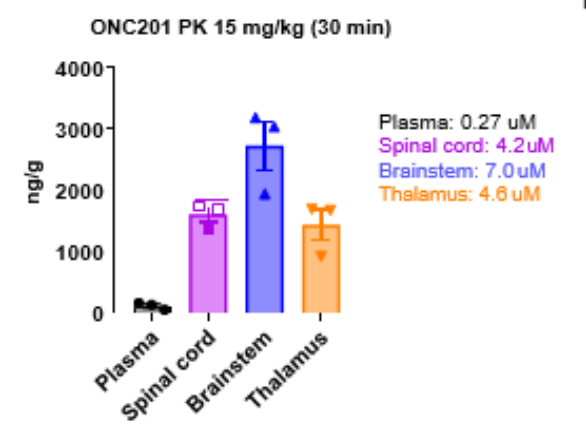

d

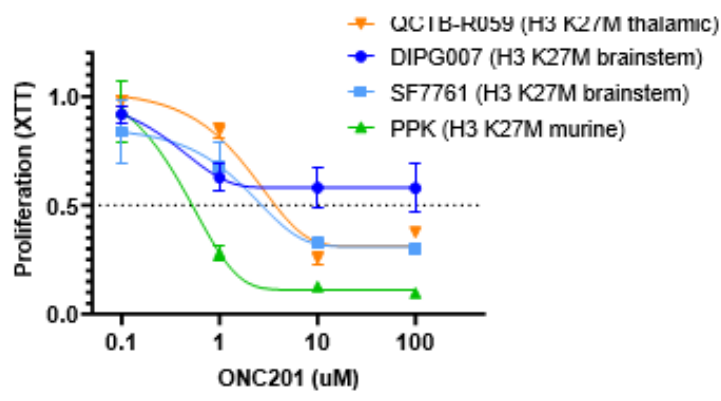

b

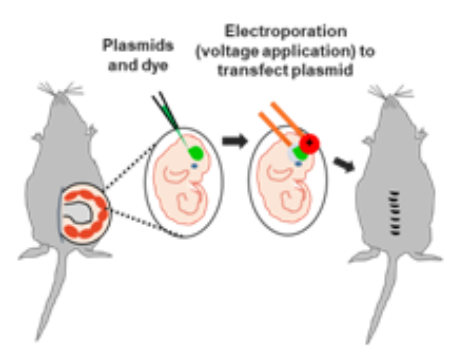

c

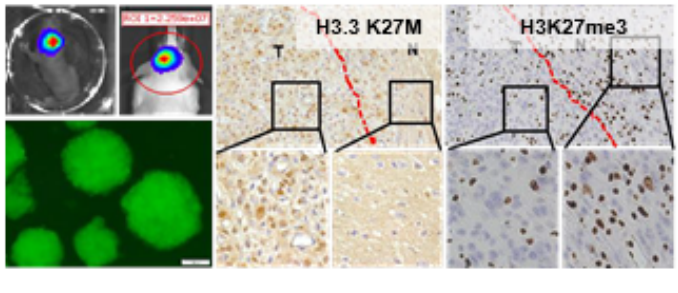

e

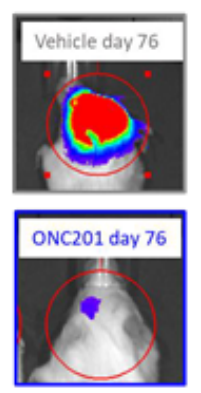

\section{Figure 1}

ONC201 demonstrates CNS penetration and survival benefit in an in utero electroporation (IUE) model. a, ONC201 preferentially accumulates in brain tissue following $15 \mathrm{mg} / \mathrm{kg}$ treatment. All brain sites show higher concentration than plasma at 30 minutes. b, Schema of IUE model used to generate H3 K27M tumors in embryonic mouse brains. c, Plasmids of mutant TP53, PDGFRA and H3 K27M mutations (PPK) 
are used to generate murine tumors that express GFP and luciferase for tumor monitoring purposes. IHC of brain tumor
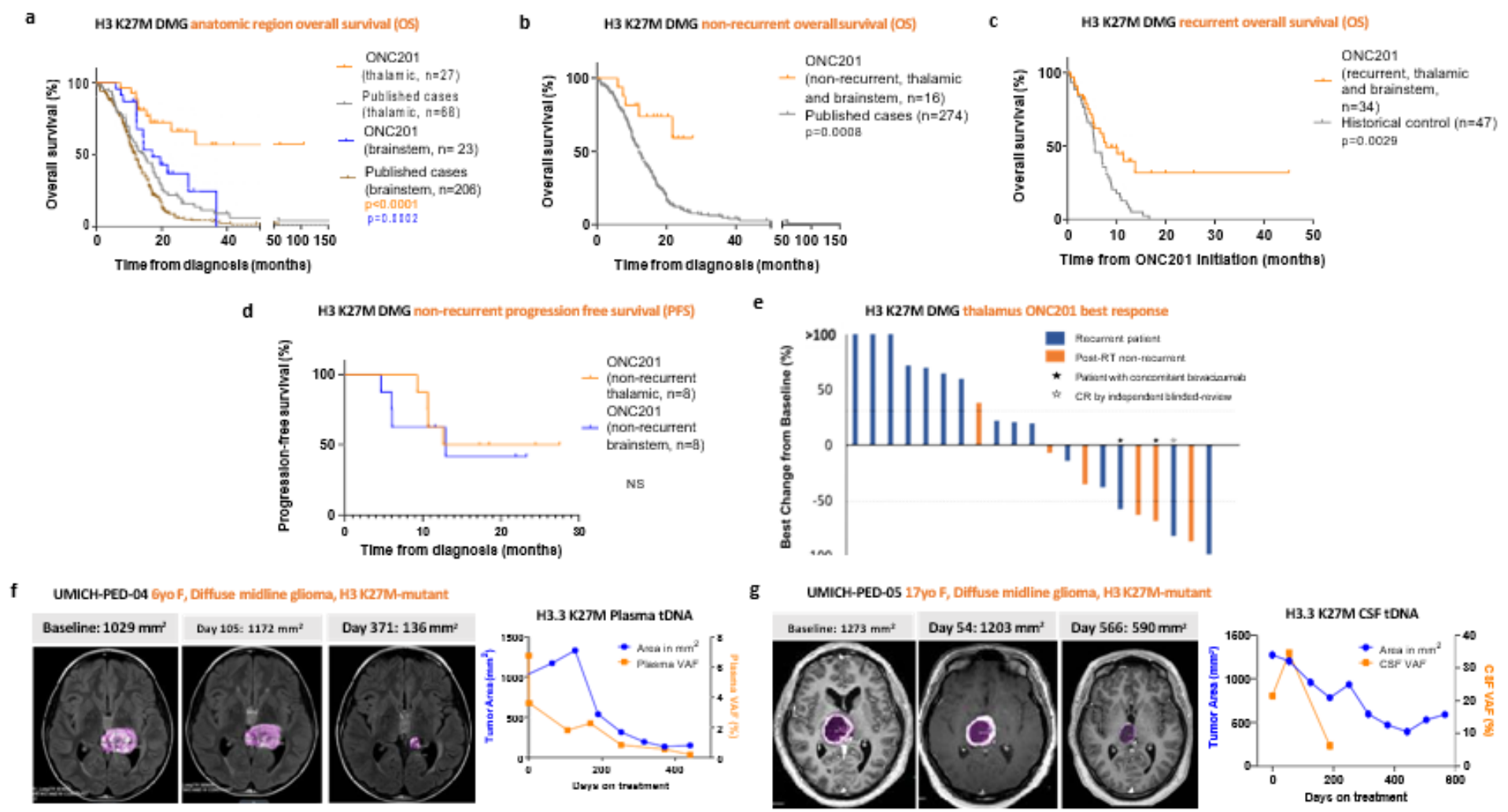

\section{Figure 2}

Survival of H3 K27M-mutant brainstem and thalamic DMG patients treated with ONC201 and H3F3A tDNA as a biomarker for ONC201 response. a, Median OS for thalamic patients has not been reached (median follow-up from diagnosis: 18.5 months). Thalamic historical median OS ( $\mathrm{n}=68$ ) is 14.5 months. Median survival for brainstem patients is 17.1 months and historical median OS ( $\mathrm{n}=206$, Pratt et al, Acta Neuropathologica, 2018) is 11.9 months. b, Median OS from diagnosis of non-recurrent thalamic and brainstem patients $(n=16)$ has not been reached (median follow-up from diagnosis: 16.8 months, median time on drug: 10.6 months). Historical median OS ( $n=274$, Pratt et al) is 12.0 months from diagnosis. $c$, Median OS from trial registration of recurrent thalamic and brainstem patients $(n=34)$ is 7.9 months (median time on drug: 3.2 months). Historical median OS of H3 K27M thalamus and brainstem DMG, $\mathrm{n}=47$ is 5.5 months from recurrence (Mackay et al, Cancer Cell, 2018 and Mueller et al, IJC, 2019). d, Median PFS is 20.1 months from diagnosis for thalamic and is 13.0 months from diagnosis for brainstem patients. e, Waterfall plot of best change from baseline in tumor burden by RANO. Twenty-one of 27 thalamic patients were evaluable and included in this analysis; dashed lines indicate $25 \%$ tumor increase (progressive disease) and 50\% tumor reduction (partial response) in tumor burden from baseline. f, UMICH-PED-04 (post-radiation, non-recurrent) experienced 87\% regression on ONC201. Serial plasma tDNA shows that H3F3A plasma tDNA correlates with radiographic response. g, For UMICH-PED05 (post-radiation, non-recurrent) , reduction of 6-month H3F3A CSF tDNA correlated with the patient's sustained and ongoing clinical response. [p value by log-rank test for all panels]. 

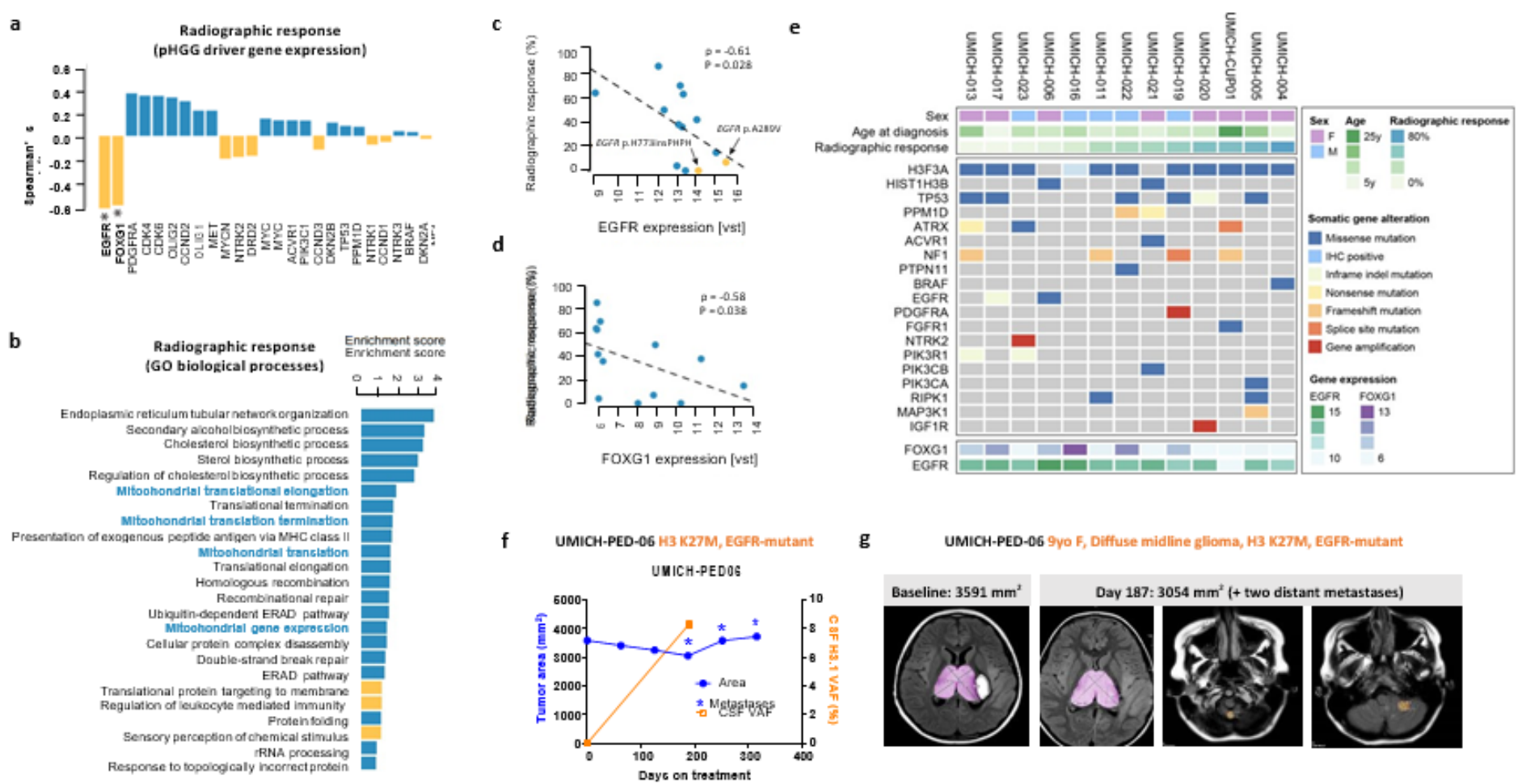

\section{Figure 3}

Elevated expression of EGFR and the cortical developmental transcription factor FOXG1 are critical negative predictors of ONC201 response in DMG. a, Correlation between best radiographic response in University of Michigan cohort $(n=13)$ and driver gene expression levels in baseline tumor RNA-seq of H3 $\mathrm{K} 27 \mathrm{M}$-mutant DMGs (yellow=negative correlation with radiographic response; blue = positive correlation; ${ }^{*} \mathrm{P}<0.05$ by algorithm AS 89). b, Gene set enrichment analysis for expression associated with radiographic response to ONC201 demonstrating positive correlation with multiple mitochondrial genesets (bold). c, Negative correlation between best radiographic response and EGFR gene expression levels in H3 K27M-mutant DMGs [variance stabilizing transformation (vst)]. d, Negative correlation between best radiographic response and FOXG1 gene expression levels in H3 K27M-mutant DMGs. e, Oncoplot for 13 thalamic and brainstem patients in UMICH cohort. f,g, UMICH-PED-06, a patient (post-radiation, nonrecurrent) with thalamic EGFR V289V mutation experienced no tumor regression and an increase in CSF tDNA at 6 months (f) on ONC201, correlating with metastatic progression (g). [Spearman's $\rho$ statistic is used to estimate a rank-based measure of association. $P$ values are computed using algorithm AS 89]. 
a

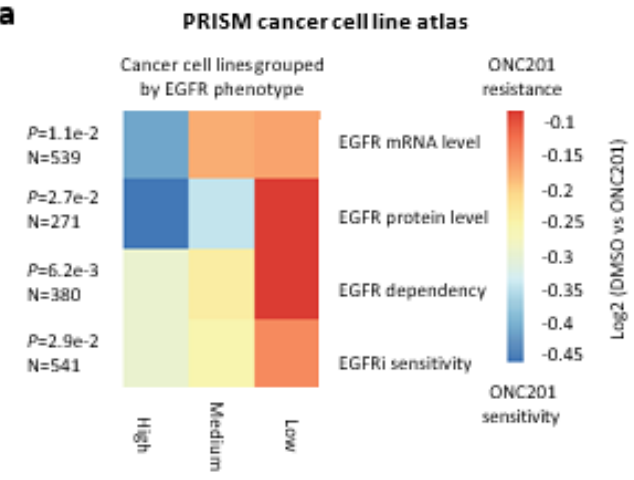

d

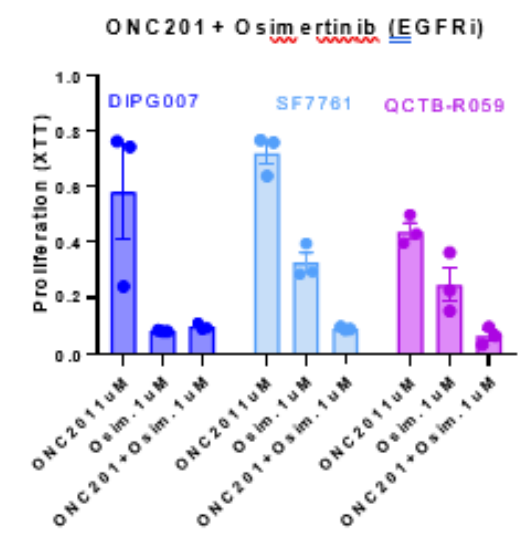

b

DIPG007 H3 K27M-mutant brainstem cell culture

c

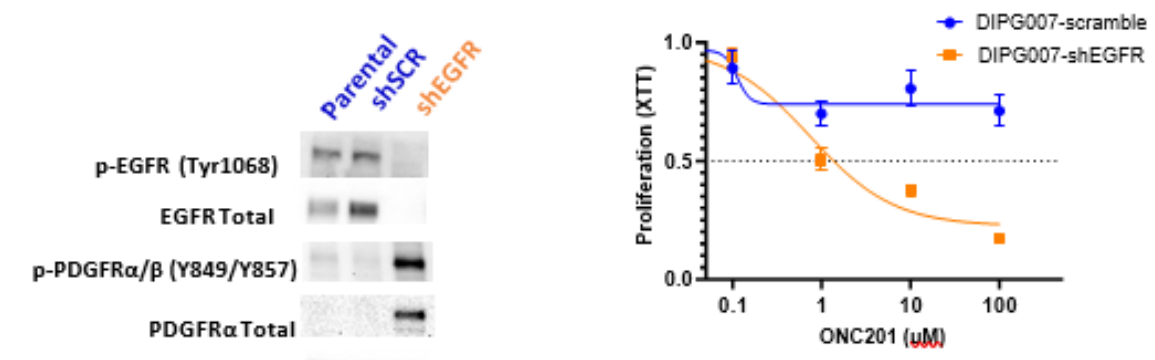

e

B-Actin

H3K27ac Chip-seq at Sox9 loci [SF7761]

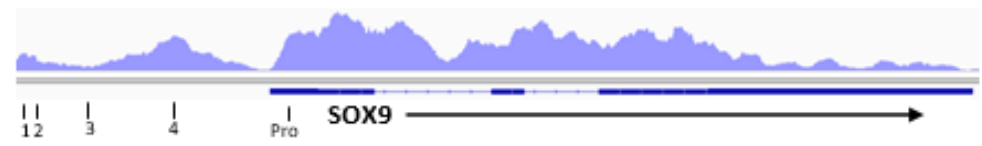

f FOXG1 Chip qPCR at Sox9 loci

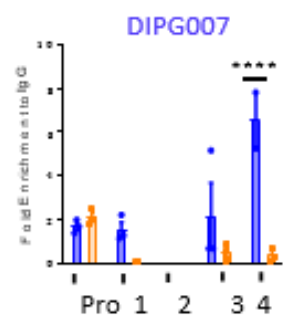

QCTB-R059
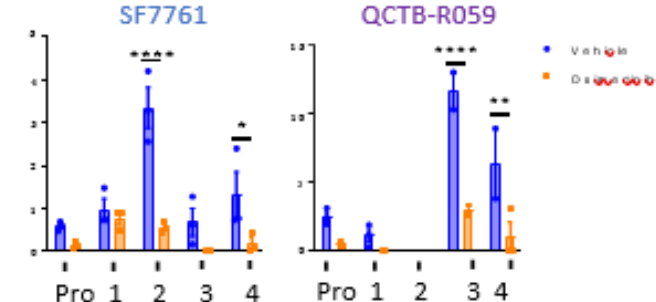

\section{Figure 4}

EGFR genetic and pharmacologic knockdown sensitizes cell lines to ONC201 treatment and reduces FOXG1 binding at established enhancer in H3 K27M cells. a, Correlation between EGFR phenotypes and ONC201 sensitivity in PRISM cancer cell lines ( $n=733)$, when organized by (i) EGFR mRNA level, (ii) EGFR protein level, (iii) EGFR CRISPR dependency, and (iv) EGFR inhibitor (EGFRi) sensitivity. b, Stable shRNA knockdown of EGFR in H3 K27M cells DIPG007 results in reduced EGFR activation and increase in PDGFRA activation. c, DIPG007-shEGFR cells demonstrate improved sensitivity to ONC201 compared to controls. d, Brainstem (SF7761) and thalamic (QCTB-R059) cell lines respond to ONC201 treatment in combination with EGFR inhibitor, osimertinib, better than either agent alone. EGFR inhibition is required to halt cell proliferation in ONC201-resistant cell line, DIPG007. e, H3K27ac ChIP-seq at SOX9 gene loci for H3 K27M SF7761 (GEO GSM2265662) displayed to denote potential enhancer ("1"- "5")/promoter sites ("PRO"). f, Chip qPCR shows reduction of FOXG1 binding (normalized to lgG) at enhancer loci with osimertinib (EGFR inhibitor) treatment across three H3 K27M primary cell cultures. [Data represent mean \pm SEM; * $\mathrm{P} \leq 0.05$, ** $\mathrm{P} \leq 0.005, * \star \star \star ~ P \leq 0.00005$ by Welch's t-test].

\section{Supplementary Files}

This is a list of supplementary files associated with this preprint. Click to download.

- SupplementaryInformation.pdf 